\title{
On aggregation of metric structures: the extended quasi-metric case
}

\author{
Sebastià Massanet, Oscar Valero \\ Department of Mathematics and Computer Science, University of the Balearic Islands \\ Campus UIB, Ctra. de Valldemossa km. 7.5 \\ Palma de Mallorca, E-07122, Spain \\ E-mail: \{s.massanet, o.valero\}@uib.es \\ Received 31 May 2012 \\ Accepted 19 August 2012
}

\begin{abstract}
In 1981, J. Borsík and J. Doboš studied and solved the problem of how to merge, by means of a function, a (not necessarily finite) collection of metrics in order to obtain a single one as output. Later on, in 2010, G. Mayor and O. Valero proposed and solved the Borsík and Doboš problem in the context of quasi-metrics. In this paper, we focus our attention on the aggregation problem for the case of extended quasi-metrics and we give several connections between both problems, the problem of merging quasi-metrics and the extended quasi-metric aggregation one.
\end{abstract}

Keywords: Aggregation, (extended) quasi-metric, monotone function, subadditive function.

\section{Introduction}

In the last years there is a growing interest in the mathematical theory of information aggregation because of its wide range of applications to practical problems. In particular for many processes that arise in applied sciences, as for instance in image processing, decision making, control theory, medical diagnosis or biology, it is necessary to process incoming data that comes from sources of a different nature in order to obtain a conclusion. In such processes the pieces of information are symbolized via some numerical values. As a consequence the fusion methods that are based on numerical aggregation operators play a central role in the theory of information aggregation. A wide class of techniques of aggregation impose a constraint in order to select the most suitable aggregation operator for the problem to be solved. In general this constraint consists of considering only those operators that provide the out- put data with the same properties as the inputs. An example of this type of situation is given when one wants to merge metrics in order to obtain a new one. Since the notion of metric plays a distinguished role in applied research, J. Borsík and J. Doboš studied the general problem of merging a collection of metrics (not necessarily finite) into a single one in ${ }^{1}$. To this end, they introduced the so-called metric aggregation functions (metric preserving functions in ${ }^{1}$ ) and characterized such functions via the notion of triangle triplets. In order to introduce the Borsík and Doboš description of the metric aggregation functions let us recall a few pertinent concepts.

We shall use the letters $\mathbb{R}, \mathbb{R}^{+}, \mathbb{N}$ to denote the set of real numbers, the set of nonnegative real numbers and the set of positive integer numbers, respectively.

From now on, $\mathbb{R}_{I}^{+}$will denote the set of all nonnegative functions defined on a (nonempty) set $I$ of indexes. Given $\mathbf{x} \in \mathbb{R}_{I}^{+}$we will write $x_{i}$ instead of $\mathbf{x}(i)$. Moreover, we will denote by $\mathbf{0}$ the element of 
$\mathbb{R}_{I}^{+}$with $0_{i}=0$ for all $i \in I$.

As usual, we will consider the set $\mathbb{R}_{I}^{+}$ordered by the pointwise order relation $\preceq$, i.e., $\mathbf{x} \preceq \mathbf{y} \Leftrightarrow x_{i} \leqslant y_{i}$ for all $i \in I$.

According to ${ }^{1}$, given $\mathbf{a}, \mathbf{b}, \mathbf{c} \in \mathbb{R}_{I}^{+}$, we will say that the triplet $(\mathbf{a}, \mathbf{b}, \mathbf{c})$ forms a triangle triplet whenever $\mathbf{a} \preceq \mathbf{b}+\mathbf{c}, \mathbf{b} \preceq \mathbf{a}+\mathbf{c}$ and $\mathbf{c} \preceq \mathbf{b}+\mathbf{a}$, i.e., whenever $a_{i} \leqslant b_{i}+c_{i}, b_{i} \leqslant a_{i}+c_{i}$ and $c_{i} \leqslant b_{i}+a_{i}$ for all $i \in I$.

A function $\Phi: \mathbb{R}_{I}^{+} \rightarrow \mathbb{R}^{+}$will be called monotone provided that $\Phi(\mathbf{x}) \leqslant \Phi(\mathbf{y})$ for all $\mathbf{x}, \mathbf{y} \in \mathbb{R}_{I}^{+}$ with $\mathbf{x} \preceq \mathbf{y}$. Furthermore, a function $\Phi: \mathbb{R}_{I}^{+} \rightarrow \mathbb{R}^{+}$ will be said to be subadditive if $\Phi(\mathbf{x}+\mathbf{y}) \leqslant \Phi(\mathbf{x})+$ $\Phi(\mathbf{y})$ for all $\mathbf{x}, \mathbf{y} \in \mathbb{R}_{I}^{+}$.

In the sequel we will denote by $\mathscr{O}_{I}$ the set of all functions $\Phi: \mathbb{R}_{I}^{+} \rightarrow \mathbb{R}^{+}$satisfying: $\Phi(\mathbf{x})=0 \Leftrightarrow \mathbf{x}=$ 0.

Following ${ }^{1}$, a function $\Phi: \mathbb{R}_{I}^{+} \rightarrow \mathbb{R}^{+}$is a metric aggregation function if the function $M_{d, \Phi}: X \times X \rightarrow$ $\mathbb{R}^{+}$is a metric for every indexed family of metric spaces $\left\{\left(X_{i}, d_{i}\right)\right\}_{i \in I}$, where $X=\prod_{i \in I} X_{i}$ and

$$
M_{d, \Phi}(x, y)=\Phi\left(\left(d_{i}\left(x_{i}, y_{i}\right)\right)_{i \in I}\right)
$$

for all $x, y \in X$.

The aforementioned characterization of metric aggregation functions can be enunciated as follows:

Theorem 1. Let $\Phi: \mathbb{R}_{I}^{+} \rightarrow \mathbb{R}^{+}$. Then the below assertions are equivalent:

(1) $\Phi$ is a metric aggregation function.

(2) $\Phi$ holds the following properties:

(2.1) $\Phi \in \mathscr{O}_{I}$.

(2.2) Let $\mathbf{a}, \mathbf{b}, \mathbf{c} \in \mathbb{R}_{I}^{+}$. If $(\mathbf{a}, \mathbf{b}, \mathbf{c})$ is a triangle triplet, then so is $(\Phi(\mathbf{a}), \Phi(\mathbf{b}), \Phi(\mathbf{c}))$.

From Theorem 1 one can easily deduce that every metric aggregation function is subadditive. In addition to the preceding relationship between metric aggregation functions and the subadditive ones, Borsík and Doboš proved the below result.

Proposition 2. Let $\Phi \in \mathscr{O}_{I}$. If $\Phi$ is monotone and subadditive, then $\Phi$ is a metric aggregation function.
On account of the above proposition and theorem, it seems natural to ask if every metric aggregation function is monotone. However, such a question has a negative answer, i.e., there are metric aggregation functions which are not monotone such as the following example shows:

Example 1. Let $I=\mathbb{N}$. Consider the function $\Phi: \mathbb{R}_{\mathbb{N}}^{+} \rightarrow \mathbb{R}^{+}$given by $\Phi(\mathbf{0})=0$ and

$$
\Phi(\mathbf{x})=\left\{\begin{array}{ll}
2 & \alpha(\mathbf{x}) \in] 0,1[ \\
1 & \alpha(\mathbf{x}) \geqslant 1
\end{array},\right.
$$

where $\mathbf{x} \neq \mathbf{0}$ and $\alpha(\mathbf{x})$ denotes the value of the first component of $\mathbf{x}$ different from 0 . Clearly $\Phi \in \mathscr{O}_{\mathbb{N}}$. Furthermore, it is easily seen that $\Phi$ turns triangle triplets into triangle triplets. Thus, by Theorem 1, $\Phi$ is a metric aggregation function. However, $\Phi$ is not a monotone function. Indeed take $\mathbf{x}, \mathbf{y} \in \mathbb{R}_{\mathbb{N}}^{+}$with $x_{i}=\frac{1}{2}, y_{i}=1$ for all $i \in \mathbb{N}$. Then $\mathbf{x} \preceq \mathbf{y}$ but $\Phi(\mathbf{x})=2$, $\Phi(\mathbf{y})=1$

Since Borsík and Doboš solved the problem of merging a collection of metrics, several authors have provided new advances in the study of the aggregation problem for several kinds of generalized metrics. Specifically E. Castiñeira, A. Pradera and E. Trillas have solved the aggregation problem for $C$-generalized metrics, $S$-generalized distances and pseudometrics in ${ }^{2},{ }^{3}$ and ${ }^{4}$, and several general techniques for merging a finite number of metrics into another one have been explored by J. Casasnovas and F. Rosselló in ${ }^{5}$ and ${ }^{6}$. In the two last references some of the obtained results have been applied to the comparison of biological sequences and to diagnosis problems in medicine.

Recently, quasi-metrics have been shown to be an appropriate tool to model several processes that arise in a natural way in Computer Science, Artificial Intelligence and Bioinformatics. In particular, an efficient framework, based on quasi-metrics, to model the running time of computing in asymptotic complexity analysis of programs and algorithms has been introduced and developed by L.M. García-Raffi, E.A. Sánchez-Pérez, S. Romaguera, M. Schellekens and O. Valero in ${ }^{7},{ }^{8},{ }^{9},{ }^{10}$ and 11. Moreover, quasi-metrics have been used successfully to describe logic programming processes by A.K. Seda in ${ }^{12}$ and ${ }^{13}$. In ${ }^{14},{ }^{15},{ }^{16}$ and ${ }^{17}$, a 
natural correspondence between similarity measures on biological (nucleotide or protein) sequences and quasi-metrics has been proved by V. Pestov, A. Stojmirović and Y.-K. Yu, giving, in addition, practical applications to search in DNA and protein datasets.

Inspired by the original work of Borsík and Doboš and motivated by the utility of quasi-metrics in the aforementioned fields of Artificial Intelligence and Computer Science, G. Mayor and O. Valero studied the aggregation problem in the quasi-metric context. Thus, they introduced the notion of quasimetric aggregation function in ${ }^{18}$ and gave characterizations of such functions in the spirit of Theorem 1 in ${ }^{18}$ and ${ }^{19}$. With the aim of presenting such characterizations let us recall some basic notions about quasi-metrics.

Following ${ }^{20}$, a quasi-metric on a (nonempty) set $X$ is a function $d: X \times X \rightarrow \mathbb{R}^{+}$such that for all $x, y, z \in X$ :

(i) $d(x, y)=d(y, x)=0 \Leftrightarrow x=y$.

(ii) $d(x, z) \leqslant d(x, y)+d(y, z)$.

Note that a metric on a set $X$ is a quasi-metric $d$ on $X$ satisfying, in addition, the following condition for all $x, y \in X$ :

(iii) $d(x, y)=d(y, x)$.

A quasi-metric space is a pair $(X, d)$ such that $X$ is a (nonempty) set and $d$ is a quasi-metric on $X$.

A well-known example of quasi-metric space is given by the pair $\left(\mathbb{R}^{+}, u\right)$ where $u$ is defined by

$$
u(x, y)=(y-x) \vee 0
$$

for all $x, y \in \mathbb{R}^{+}$.

If $d$ is a quasi-metric on $X$, then the function $d^{-1}: X \times X \rightarrow \mathbb{R}^{+}$defined for all $x, y \in X$ by

$$
d^{-1}(x, y)=d(y, x)
$$

is again a quasi-metric, called the conjugate of $d$. Note that each quasi-metric $d$ induces a metric $d^{s}$ on $X \times X$ as follows:

$$
d^{S}(x, y)=d(x, y) \vee d^{-1}(x, y)
$$

for all $x, y \in X$, where we denote by $\vee$ the maximum operator.
According to ${ }^{18}$ and ${ }^{19}$, a function $\Phi: \mathbb{R}_{I}^{+} \rightarrow \mathbb{R}^{+}$ is a quasi-metric aggregation function if the function $Q_{d, \Phi}: X \times X \rightarrow \mathbb{R}^{+}$is a quasi-metric for every indexed family of quasi-metric spaces $\left\{\left(X_{i}, d_{i}\right)\right\}_{i \in I}$, where $X=\prod_{i \in I} X_{i}$ and

$$
Q_{d, \Phi}(x, y)=\Phi\left(\left(d_{i}\left(x_{i}, y_{i}\right)\right)_{i \in I}\right)
$$

for all $x, y \in X$.

The quasi-metric formulation of Theorem 1, given in ${ }^{19}$, can be stated in the following way:

Theorem 3. Let $\Phi: \mathbb{R}_{I}^{+} \rightarrow \mathbb{R}^{+}$. Then the below assertions are equivalent:

(1) $\Phi$ is a quasi-metric aggregation function.

(2) $\Phi$ holds the following properties:

(2.1) $\Phi \in \mathscr{O}_{I}$

(2.2) Let $\mathbf{a}, \mathbf{b}, \mathbf{c} \in \mathbb{R}_{I}^{+}$. If $\mathbf{a} \preceq \mathbf{b}+\mathbf{c}$, then $\Phi(\mathbf{a}) \leqslant$ $\Phi(\mathbf{b})+\Phi(\mathbf{c})$.

Of course from the preceding result, and by Theorem 1 , one can deduce immediately that every quasi-metric aggregation function is a metric aggregation one. The converse is not true in general such as Example 1 shows. Moreover, Theorem 3 yields that every quasi-metric aggregation function is, similarly to the metric case, subadditive. Furthermore, notice that, contrary to the metric case, the preceding characterization states that every quasi-metric aggregation function is always a monotone function. In fact, as a consequence of Theorem 3, we have the next characterization of quasi-metric aggregation function which was obtained in ${ }^{18}$.

Theorem 4. Let $\Phi: \mathbb{R}_{I}^{+} \rightarrow \mathbb{R}^{+}$. Then the below assertions are equivalent:

(1) $\Phi$ is a quasi-metric aggregation function

(2) $\Phi$ is a subadditive and monotone function such that $\Phi \in \mathscr{O}_{I}$.

The fact that, from a given quasi-metric $d$, one can always generate the conjugate quasi-metric $d^{-1}$ induces to wonder what is the relationship between the conjugate quasi-metric induced by the quasimetric obtained via the aggregation of an indexed family of quasi-metrics and the quasi-metric generated by the aggregation of the conjugate quasimetrics associated to the aforesaid family. The next 
result, which was given in ${ }^{19}$, provides the answer to the formulated question.

Proposition 5. Let $\Phi: \mathbb{R}_{I}^{+} \rightarrow \mathbb{R}^{+}$be a quasi-metric aggregation function and let $\left\{\left(X_{i}, d_{i}\right)\right\}_{i \in I}$ be a family of quasi-metric spaces. Then

$$
Q_{d, \Phi}^{-1}(x, y)=Q_{d^{-1}, \Phi}(x, y)
$$

for all $x, y \in X=\prod_{i \in I} X_{i}$, where

$$
Q_{d^{-1}, \Phi}(x, y)=\Phi\left(\left(d_{i}^{-1}\left(x_{i}, y_{i}\right)\right)_{i \in I}\right)
$$

for all $x, y \in X$.

Since every quasi-metric $d$ induces a metric $d^{s}$ it also seems natural to wonder what is the relationship between the metric induced by the quasi-metric generated via the aggregation of an indexed family of quasi-metrics and the metric generated through the aggregation of the family of metrics associated to each quasi-metric of the aforesaid family. The answer to the posed question was given again in ${ }^{19}$ and is provided by the below result.

Proposition 6. Let $\Phi: \mathbb{R}_{I}^{+} \rightarrow \mathbb{R}^{+}$be a quasi-metric aggregation function and let $\left\{\left(X_{i}, d_{i}\right)\right\}_{i \in I}$ be a family of quasi-metric spaces. Then

$$
Q_{d, \Phi}^{s}(x, y) \leqslant Q_{d^{s}, \Phi}(x, y) \leqslant 2 Q_{d, \Phi}^{s}(x, y)
$$

for all $x, y \in X=\prod_{i \in I} X_{i}$, where

$$
Q_{d^{s}, \Phi}(x, y)=\Phi\left(\left(d_{i}^{s}\left(x_{i}, y_{i}\right)\right)_{i \in I}\right)
$$

for all $x, y \in X$.

Observe that in the statement of the preceding result the function $Q_{d^{s}, \Phi}$ is a metric, since every quasimetric aggregation function is a metric aggregation function.

Prompted by the work of Rosselló and Casasnovas developed in ${ }^{5},{ }^{6}$, Casasnovas and Valero, and P. Tirado and Valero obtained several connections between the quasi-metric aggregation problem and the asymptotic computational complexity theory in ${ }^{21}$ and ${ }^{22}$, respectively. Moreover, some other problems related to the quasi-metric aggregation one have been tackle by S. Massanet and Valero in ${ }^{23}$, and by J. Martín, Mayor and Valero in ${ }^{24}$ and ${ }^{25}$.
Motivated, on the one hand, by the recent advances in the study of the quasi-metric aggregation problem and, on the other hand, by the utility of the so-called extended quasi-metrics in Computer Science (see, for instance, ${ }^{10},{ }^{26}$ and ${ }^{27}$ ), in this paper we focus our attention on the aggregation problem for the case of the aforementioned extended quasimetrics. Thus, we introduce the notion of extended quasi-metric aggregation function and we provide a description of such functions. In addition, we connect the problem of merging quasi-metrics with the extended quasi-metric aggregation one.

\section{The Extended Quasi-metric Aggregation Problem}

In the remainder of the paper we will denote by $\mathbb{R}_{\infty}^{+}$ the set $\mathbb{R}^{+} \cup\{\infty\}$. Moreover, we will consider the set $\mathbb{R}_{\infty}^{+}$endowed with the order relation $\leqslant_{\infty}$ given by $x \leqslant \infty y \Leftrightarrow y=\infty$ and $x \in \mathbb{R}_{\infty}^{+}$or $x, y \in \mathbb{R}^{+}$with $x \leqslant y$.

Of course we will write $x<_{\infty} y \Leftrightarrow x \leqslant_{\infty} y$ and $x \neq y$.

Let us recall that an extended quasi-metric on a (nonempty) set $X$ is a function $d: X \times X \rightarrow \mathbb{R}_{\infty}^{+}$such that for all $x, y, z \in X$ :

(i) $d(x, y)=d(y, x)=0 \Leftrightarrow x=y$.

(ii) $d(x, z) \leqslant_{\infty} d(x, y)+d(y, z)$.

Note that an extended quasi-metric satisfies the same axioms as the quasi-metrics except that $d(x, y)=\infty$ is allowed. Moreover, every quasimetric is an extended quasi-metric.

Clearly an extended metric on a set $X$ is an extended quasi-metric $d$ on $X$ satisfying for all $x, y \in$ $X$ :

(iii) $d(x, y)=d(y, x)$.

Naturally, an extended quasi-metric space is a pair $(X, d)$ such that $X$ is a (nonempty) set and $d$ is an extended quasi-metric on $X$.

A well-known example of extended quasi-metric space, which is not a quasi-metric space, is given by the pair $\left(\mathbb{R}^{+}, d_{S}\right)$, where

$$
d_{S}(x, y)=\left\{\begin{array}{ll}
y-x & \text { if } x \leqslant y \\
\infty & \text { if } x>y
\end{array} .\right.
$$


Similar to the case of quasi-metrics, when $d$ is an extended quasi-metric on $X$, then the function $d^{-1}: X \times X \rightarrow \mathbb{R}_{\infty}^{+}$defined for all $x, y \in X$ by

$$
d^{-1}(x, y)=d(y, x)
$$

is again an extended quasi-metric, that we will call, again, the conjugate of $d$. Moreover, each extended quasi-metric $d$ induces an extended metric $d^{s}$ on $X \times X$ given, for all $x, y \in X$, by

$$
d^{s}(x, y)=d(x, y) \vee_{\infty} d^{-1}(x, y),
$$

where $V_{\infty}$ stands for the maximum operator with respect to the order $\leqslant_{\infty}$.

In order to tackle the aggregation problem for the case of extended quasi-metrics we fix some terminology as follows:

We will consider the set of all functions defined from a (nonempty) set of indexes $I$ into $\mathbb{R}_{\infty}^{+}$and we will denote it by $\mathbb{R}_{I, \infty}^{+}$. Of course, given $\mathbf{x} \in \mathbb{R}_{I, \infty}^{+}$we will write $x_{i}$ instead of $\mathbf{x}(i)$. Furthermore, we will consider the set $\mathbb{R}_{I, \infty}^{+}$ordered by the pointwise order relation $\preceq_{\infty}$, i.e., $\mathbf{x} \preceq_{\infty} \mathbf{y} \Leftrightarrow x_{i} \leqslant_{\infty} y_{i}$ for all $i \in I$.

In the light of the preceding notions we are able to introduce the concept of extended quasi-metric aggregation function.

Definition 1. A function $\Phi: \mathbb{R}_{I, \infty}^{+} \rightarrow \mathbb{R}_{\infty}^{+}$is an extended quasi-metric aggregation function if the function $E Q_{d, \Phi}: X \times X \rightarrow \mathbb{R}_{\infty}^{+}$is an extended quasimetric for every indexed family of extended quasimetric spaces $\left\{\left(X_{i}, d_{i}\right)\right\}_{i \in I}$, where $X=\prod_{i \in I} X_{i}$ and

$$
E Q_{d, \Phi}(x, y)=\Phi\left(\left(d_{i}\left(x_{i}, y_{i}\right)\right)_{i \in I}\right)
$$

for all $x, y \in X$.

Now we focus our investigation on the study of a characterization in the spirit of Theorems 3 and 4 . To this end, we will denote by $\mathscr{O}_{I}^{\infty}$ the set of all functions $\Phi: \mathbb{R}_{I, \infty}^{+} \rightarrow \mathbb{R}_{\infty}^{+}$satisfying: $\Phi(\mathbf{x})=0 \Leftrightarrow \mathbf{x}=\mathbf{0}$, and we will say that a function $\Phi: \mathbb{R}_{I, \infty}^{+} \rightarrow \mathbb{R}_{\infty}^{+}$ is monotone (subadditive) provided that $\Phi(\mathbf{x}) \leqslant \infty$ $\Phi(\mathbf{y})$ for all $\mathbf{x}, \mathbf{y} \in \mathbb{R}_{I, \infty}^{+}$with $\mathbf{x} \preceq_{\infty} \mathbf{y}\left(\Phi(\mathbf{x}+\mathbf{y}) \leqslant_{\infty}\right.$ $\Phi(\mathbf{x})+\Phi(\mathbf{y})$ for all $\left.\mathbf{x}, \mathbf{y} \in \mathbb{R}_{I, \infty}^{+}\right)$. Of course, we make the convention that $\mathbf{a}+\infty=\infty$ for all $\mathbf{a} \in \mathbb{R}_{\infty}^{+}$.

The next result allows to provide examples of extended quasi-metric aggregation functions.
Proposition 7. Let $\Phi \in \mathscr{O}_{I}^{\infty}$. If $\Phi$ is monotone and subadditive, then $\Phi$ is an extended quasi-metric preserving function.

Proof. Given an indexed family of extended quasimetric spaces $\left\{\left(X_{i}, d_{i}\right)\right\}_{i \in I}$ we have to show that the function $E Q_{d, \Phi}$ in Definition 1 is an extended quasimetric. With this aim, assume that

$$
E Q_{d, \Phi}(x, y)=E Q_{d, \Phi}(y, x)=0
$$

for some $x, y \in X=\prod_{i \in I} X_{i}$. Then we deduce that $d_{i}\left(x_{i}, y_{i}\right)=d_{i}\left(y_{i}, x_{i}\right)=0$ for all $i \in I$, since $\Phi \in \mathscr{O}_{I}^{\infty}$. From the fact that every $d_{i}$ is an extended quasimetric we obtain that $x_{i}=y_{i}$ for all $i \in I$ and, thus, that $x=y$. It is clear that $E Q_{d, \Phi}(x, x)=0$ for all $x \in X$.

Next we prove that $E Q_{d, \Phi}(x, y) \leqslant \infty$ $E Q_{d, \Phi}(x, z)+E Q_{d, \Phi}(z, y)$ for all $x, y, z \in X$.

Let $x, y, z \in X$. Since each $d_{i}$ is an extended quasimetric we have that

$$
d_{i}\left(x_{i}, y_{i}\right) \leqslant \infty d_{i}\left(x_{i}, z_{i}\right)+d_{i}\left(z_{i}, y_{i}\right)
$$

for all $i \in I$.

On the one hand, the monotonicity of $\Phi$ provides that

$$
\Phi\left(\left(d_{i}\left(x_{i}, y_{i}\right)\right)_{i \in I}\right) \leqslant \infty \Phi\left(\left(d_{i}\left(x_{i}, z_{i}\right)+d_{i}\left(z_{i}, y_{i}\right)\right)_{i \in I}\right) .
$$

On the other hand, the fact that $\Phi$ is subadditive gives that

$$
\begin{aligned}
\Phi\left(\left(d_{i}\left(x_{i}, z_{i}\right)+d_{i}\left(z_{i}, y_{i}\right)\right)_{i \in I}\right) \leqslant_{\infty} & \left.\Phi\left(\left(d_{i}\left(x_{i}, z_{i}\right)\right)\right)_{i \in I}\right) \\
+ & \left.\Phi\left(\left(d_{i}\left(z_{i}, y_{i}\right)\right)\right)_{i \in I}\right) .
\end{aligned}
$$

Combining the preceding inequalities we deduce that

$$
\begin{aligned}
E Q_{d, \Phi}(x, y) & =\Phi\left(\left(d_{i}\left(x_{i}, y_{i}\right)\right)_{i \in I}\right) \\
& \left.\left.\leqslant \infty\left(\left(d_{i}\left(x_{i}, z_{i}\right)\right)\right)_{i \in I}\right)+\Phi\left(\left(d_{i}\left(z_{i}, y_{i}\right)\right)\right)_{i \in I}\right) \\
& =E Q_{d, \Phi}(x, z)+E Q_{d, \Phi}(z, y) .
\end{aligned}
$$

So we have proved that the function $\Phi$ is exactly an extended quasi-metric aggregation function as claimed.

Next we give an example of extended quasimetric aggregation function. 
Example 2. Set $I=\{1\}$. Consider the function $\Phi: \mathbb{R}_{I, \infty}^{+} \rightarrow \mathbb{R}_{\infty}^{+}$defined by

$$
\Phi(\mathbf{x})=\left\{\begin{array}{ll}
\infty & \text { if } \mathbf{x} \neq 0 \\
0 & \text { if } \mathbf{x}=0
\end{array} .\right.
$$

It is obvious that $\Phi \in \mathscr{O}_{I}^{\infty}$ and that $\Phi$ is monotone and subadditive. So, by Proposition 7, we have that $\Phi$ is an extended quasi-metric aggregation function.

The following remark and lemma will allow us to provide a description of the extended quasi-metric aggregation functions.

Remark 1. Set $\left(\mathbb{R}_{\infty}^{+}\right)^{2}=\mathbb{R}_{\infty}^{+} \times \mathbb{R}_{\infty}^{+}$. Let $u_{\infty}: \mathbb{R}_{\infty}^{+} \times$ $\mathbb{R}_{\infty}^{+} \rightarrow \mathbb{R}_{\infty}^{+}$be the extended quasi-metric given by

$$
u_{\infty}(x, y)= \begin{cases}\infty & \text { if } x<_{\infty} y \text { and } y=\infty \\ u(x, y) & \text { if } x, y \in \mathbb{R}^{+} \\ 0 & \text { if } x=\infty \text { and } y \in \mathbb{R}^{+}\end{cases}
$$

for all $x, y \in \mathbb{R}_{\infty}^{+}$and $u$ is the quasi-metric given in Equation (1). Define the extended quasi-metric $U_{\infty}$ on $\left(\mathbb{R}_{\infty}^{+}\right)^{2}$ by

$$
U_{\infty}(x, y)=u_{\infty}\left(x_{1}, y_{1}\right)+u_{\infty}\left(x_{2}, y_{2}\right)
$$

for all $x=\left(x_{1}, x_{2}\right), y=\left(y_{1}, y_{2}\right) \in\left(\mathbb{R}_{\infty}^{+}\right)^{2}$. Then it is easily seen that for every $a, b \in \mathbb{R}_{\infty}^{+}$

$$
U_{\infty}\left(x_{a b}, y_{a b}\right)=a \text { and } U_{\infty}^{-1}\left(x_{a b}, y_{a b}\right)=b,
$$

where $x_{a b}=(0, b)$ and $y_{a b}=(a, 0)$. Observe that if $a=b$, then

$$
U_{\infty}\left(x_{a}, y_{a}\right)=a=U_{\infty}^{-1}\left(x_{a}, y_{a}\right),
$$

with $x_{a}=(0, a)$ and $y_{a}=(a, 0)$.

In this lemma, we will use the extended quasimetric $d_{S}$ introduced in Equation (2).

Lemma 8. Let $a, b \in \mathbb{R}_{\infty}^{+}$. Then there always exist $u_{a b}, v_{a b}, w_{a b} \in \mathbb{R}^{+}$such that $d_{S}\left(u_{a b}, v_{a b}\right)=a+b$, $d_{S}\left(u_{a b}, w_{a b}\right)=a$ and $d_{S}\left(w_{a b}, v_{a b}\right)=b$.

Proof. Fix $a, b \in \mathbb{R}_{\infty}^{+}$. We distinguish four possible cases.

Case 1. $a+b<\infty$. Then $a, b<\infty$ Put $u_{a b}=0, v_{a b}=a+b$ and $w_{a b}=a$. It follows that $d_{S}\left(u_{a b}, v_{a b}\right)=a+b, d_{S}\left(u_{a b}, w_{a b}\right)=a$ and that $d_{S}\left(w_{a b}, v_{a b}\right)=b$.
Case 2. $a+b=\infty$ with $a=\infty$ and $b<\infty$. Put $u_{a b}=b+1, v_{a b}=b$ and $w_{a b}=0$. It follows that $d_{S}\left(u_{a b}, v_{a b}\right)=\infty, d_{S}\left(u_{a b}, w_{a b}\right)=\infty$ and $d_{S}\left(w_{a b}, v_{a b}\right)=b$.

Case 3. $a+b=\infty$ with $a<\infty$ and $b=\infty$. Put $u_{a b}=a+1, v_{a b}=\frac{a+1}{2}$ and $w_{a b}=2 a+1$. It follows that $d_{S}\left(u_{a b}, v_{a b}\right)=\infty, d_{S}\left(u_{a b}, w_{a b}\right)=a$ and $d_{S}\left(w_{a b}, v_{a b}\right)=\infty$.

Case 4. $a+b=\infty$ with $a=b=\infty$. Put $u_{a b}=2$, $v_{a b}=0$ and $w_{a b}=1$. It follows that $d_{S}\left(u_{a b}, v_{a b}\right)=$ $d_{S}\left(u_{a b}, w_{a b}\right)=d_{S}\left(w_{a b}, v_{a b}\right)=\infty$.

In the next results we describe extended quasimetric aggregation functions.

Theorem 9. Let $\Phi: \mathbb{R}_{I, \infty}^{+} \rightarrow \mathbb{R}_{\infty}^{+}$. Then, among the below assertions, $(1) \rightarrow(2)$ :

(1) $\Phi$ is an extended quasi-metric aggregation function.

(2) $\Phi \in \mathscr{O}_{I}^{\infty}$ and $\Phi$ is subadditive.

Proof. First we prove that $\Phi \in \mathscr{O}_{I}^{\infty}$. Indeed, let $\mathbf{x} \in \mathbb{R}_{I, \infty}^{+}$such that $\Phi(\mathbf{x})=0$. Take the indexed family of extended quasi-metric spaces $\left\{\left(X_{i}, d_{i}\right)\right\}_{i \in I}$ with $X_{i}=\left(\mathbb{R}_{\infty}^{+}\right)^{2}$ and $d_{i}=U_{\infty}$ for all $i \in I$. Since $x_{i} \in \mathbb{R}_{\infty}^{+}$ for all $i \in I$, we have, by Remark 1 that $U_{\infty}\left(x_{x_{i}}, y_{x_{i}}\right)=$ $U_{\infty}^{-1}\left(x_{x_{i}}, y_{x_{i}}\right)=x_{i}$ for all $i \in I$, where $x_{x_{i}}=\left(0, x_{i}\right)$ and $y_{x_{i}}=\left(x_{i}, 0\right)$ for all $i \in I$. Take $x, y \in \prod_{i \in I}\left(\mathbb{R}_{\infty}^{+}\right)^{2}$ such that $x_{i}=x_{x_{i}}$ and $y_{i}=y_{x_{i}}$. Hence

$$
E Q_{d, \Phi}(x, y)=\Phi\left(\left(U_{\infty}\left(x_{x_{i}}, y_{x_{i}}\right)_{i \in I}\right)=\Phi(\mathbf{x})=0\right.
$$

and

$$
E Q_{d, \Phi}(y, x)=\Phi\left(\left(U_{\infty}\left(y_{x_{i}}, x_{x_{i}}\right)_{i \in I}\right)=\Phi(\mathbf{x})=0 .\right.
$$

The fact that $\Phi$ is an extended quasi-metric aggregation function yields that $E Q_{d, \Phi}$ is an extended quasi-metric and, thus, that $x=y$. Whence we deduce that $\mathbf{x}=\mathbf{0}$ and, thus that $\Phi \in \mathscr{O}_{I}^{\infty}$.

Next we show that $\Phi$ is subadditive. To this end, let $\mathbf{a}, \mathbf{b} \in \mathbb{R}_{I, \infty}^{+}$.

By Lemma 8 there exists $u_{a_{i} b_{i}}, v_{a_{i} b_{i}}, w_{a_{i} b_{i}} \in \mathbb{R}^{+}$ such that $d_{S}\left(u_{a_{i} b_{i}}, v_{a_{i} b_{i}}\right)=a_{i}+b_{i}, d_{S}\left(u_{a_{i} b_{i}}, w_{a_{i} b_{i}}\right)=a_{i}$ and $d_{S}\left(w_{a_{i} b_{i}}, v_{a_{i} b_{i}}\right)=b_{i}$ for all $i \in I$. 
Set $u_{a b}, v_{a b}, w_{a b}$ the elements of $\mathbb{R}_{I, \infty}^{+}$such that $u_{a b_{i}}=u_{a_{i} b_{i}}, v_{a b_{i}}=v_{a_{i} b_{i}}$ and $w_{a b_{i}}=w_{a_{i} b_{i}}$ for all $i \in I$.

Consider the indexed family of extended quasimetric spaces $\left\{\left(X_{i}, d_{i}\right)\right\}_{i \in I}$ with $X_{i}=\mathbb{R}^{+}$and $d_{i}=d_{S}$ for all $i \in I$. Since $\Phi$ is an extended quasi-metric aggregation function we have that

$$
\begin{aligned}
\Phi(\mathbf{a}+\mathbf{b}) & =\Phi\left(\left(d_{S}\left(u_{a_{i} b_{i}}, v_{a_{i} b_{i}}\right)_{i \in I}\right)\right. \\
& =E Q_{d, \Phi}\left(u_{a b}, v_{a b}\right) \\
& \leqslant \\
& =\Phi Q_{d, \Phi}\left(u_{a b}, w_{a b}\right)+E Q_{d, \Phi}\left(w_{a b}, v_{a b}\right) \\
& +\Phi\left(d_{S}\left(u_{a_{i} b_{i}}, w_{a_{i} b_{i}}\right)_{i \in I}\right) \\
& =\Phi(\mathbf{a})+\Phi(\mathbf{b}) .
\end{aligned}
$$

Remark 2. Let us recall that a (n extended) quasimetric $d$ on a nonempty set $X$ is called $T_{1}$ if given $x, y \in X$ with $d(x, y)=0 \Rightarrow x=y$. On account of the proof of Theorem 9, it is clear that $E Q_{d, \Phi}$ is a $T_{1}$ extended quasi-metric on $X=\prod_{i \in I} X_{i}$ whenever the extended quasi-metric aggregation function operates on a family of extended quasi-metric spaces $\left\{\left(X_{i}, d_{i}\right)\right\}_{i \in I}$ such that $d_{i}$ is $T_{1}$ for all $i \in I$.

In the light of Theorem 4 , it seems natural to discuss if, similar to the quasi-metric case, the extended quasi-metric aggregation functions are exactly those that satisfy all requirements in statement (2) in Theorem 9 and, in addition, the monotonicity. Nevertheless, the below example shows that there are extended quasi-metric aggregation functions which are not monotone.

Example 3. Let $I=\{1,2\}$. Consider the function $\Phi: \mathbb{R}_{I, \infty}^{+} \rightarrow \mathbb{R}_{\infty}^{+}$defined by

$$
\Phi(\mathbf{x})= \begin{cases}\infty & x_{1}>0 \\ 2 & \left.x_{1}=0, x_{2} \in\right] 0,1[ \\ 1 & x_{1}=0, x_{2} \geqslant 1 \\ 0 & x_{1}=x_{2}=0\end{cases}
$$

for all $\mathbf{x}=\left(x_{1}, x_{2}\right) \in \mathbb{R}_{I, \infty}^{+}$.

It is evident that $\Phi \in \mathscr{O}_{I}^{\infty}$. It is a simple matter to check that $\Phi$ is subadditive. Moreover, it is clear that $\left(0, \frac{1}{2}\right) \preceq_{\infty}(0,1)$ but $\Phi\left(0, \frac{1}{2}\right)=2$ and $\Phi(0,1)=1$. So $\Phi$ is not monotone. Howeover, $\Phi$ is an extended quasi-metric aggregation function.
Although the preceding example shows that a version of Theorem 4 does not hold in the extended quasi-metric context, we have the following result.

Theorem 10. Let $\Phi: \mathbb{R}_{I, \infty}^{+} \rightarrow \mathbb{R}_{\infty}^{+}$. Then, among the below assertions, $(2) \rightarrow(1)$ :

(1) $\Phi$ is an extended quasi-metric aggregation function.

(2) $\Phi$ holds the following properties:

(2.1) $\Phi \in \mathscr{O}_{I}^{\infty}$.

(2.2) Let $\boldsymbol{a}, \boldsymbol{b}, \boldsymbol{c} \in \mathbb{R}_{I, \infty}^{+}$. If $\boldsymbol{a} \preceq_{\infty} \boldsymbol{b}+\boldsymbol{c}$, then $\Phi(\boldsymbol{a}) \leqslant_{\infty}$ $\Phi(\boldsymbol{b})+\Phi(\boldsymbol{c})$.

Proof. It is clear, from condition (2.2), that $\Phi$ is subadditive since $\mathbf{a}+\mathbf{b} \preceq_{\infty} \mathbf{a}+\mathbf{b}$ for all $\mathbf{a}, \mathbf{b} \in \mathbb{R}_{I, \infty}^{+}$. Let $\mathbf{a}, \mathbf{b} \in \mathbb{R}_{I, \infty}^{+}$be such that $\mathbf{a} \preceq_{\infty} \mathbf{b}$. Then taking $\mathbf{c}=\mathbf{0}$ in condition (2.2) we have that $\Phi(\mathbf{a}) \leqslant \infty$ $\Phi(\mathbf{b})+\Phi(\mathbf{0})$. Since condition (2.1) guarantees that $\Phi(\mathbf{0})=0$ we deduce that $\Phi(\mathbf{a}) \leqslant_{\infty} \Phi(\mathbf{b})$ and, hence, that $\Phi$ is monotone. Therefore, by Proposition 7, we conclude that $\Phi$ is an extended quasi-metric aggregation function.

Observe that Example 3 shows that, in the preceding theorem, (1) $\rightarrow$ (2) does not hold in general because every extended quasi-metric aggregation function satisfying condition (2.2) in statement (2) in Theorem 10 is monotone.

Next we provide a characterization of the extended quasi-metric aggregation functions which satisfy condition (2.2) in statement of Theorem 10.

Theorem 11. Let $\Phi: \mathbb{R}_{I, \infty}^{+} \rightarrow \mathbb{R}_{\infty}^{+}$be an extended quasi-metric aggregation function. Then the following assertions are equivalent:

(1) $\Phi$ holds condition (2.2) in statement of Theorem 10.

(2) $\Phi$ is monotone.

Proof. (1) $\rightarrow$ (2). Taking $\mathbf{c}=\mathbf{0}$ we obtain that $\Phi(\mathbf{a}) \leqslant_{\infty} \Phi(\mathbf{b})+\Phi(\mathbf{0})$ whenever $\mathbf{a} \preceq_{\infty} \mathbf{b}+\mathbf{0}$. By statement (2) in Theorem 9 we deduce that $\Phi(\mathbf{0})=0$ and, thus, that $\Phi(\mathbf{a}) \leqslant_{\infty} \Phi(\mathbf{b})$.

$(2) \rightarrow(1)$. Let $\mathbf{a}, \mathbf{b}, \mathbf{c} \in \mathbb{R}_{I, \infty}^{+}$such that $\mathbf{a} \preceq_{\infty}$ $\mathbf{b}+\mathbf{c}$. Then $\Phi(\mathbf{a}) \leqslant_{\infty} \Phi(\mathbf{b}+\mathbf{c})$, since $\Phi$ is monotone. 
Moreover, Theorem 9 guarantees that $\Phi$ is subadditive. Whence we deduce that $\Phi(\mathbf{a}) \leqslant_{\infty} \Phi(\mathbf{b})+\Phi(\mathbf{c})$.

The next result is a version of Proposition 5 in the framework of extended quasi-metric aggregation functions. We include its proof for the sake of completeness although it runs following exactly the same argument to those given in the proof of Proposition 5 in ${ }^{19}$.

Proposition 12. Let $\Phi: \mathbb{R}_{I, \infty}^{+} \rightarrow \mathbb{R}_{\infty}^{+}$be an extended quasi-metric aggregation function and let $\left\{\left(X_{i}, d_{i}\right)\right\}_{i \in I}$ be an indexed family of extended quasimetric spaces. Then

$$
E Q_{d, \Phi}^{-1}(x, y)=E Q_{d^{-1}, \Phi}(x, y)
$$

for all $x, y \in X=\prod_{i \in I} X_{i}$, where

$$
E Q_{d^{-1}, \Phi}(x, y)=\Phi\left(\left(d_{i}^{-1}\left(x_{i}, y_{i}\right)\right)_{i \in I}\right)
$$

for all $x, y \in X$.

Proof. Let $x, y \in X=\prod_{i \in I} X_{i}$. On the one hand,

$$
E Q_{d, \Phi}^{-1}(x, y)=E Q_{d, \Phi}(y, x)=\Phi\left(\left(d_{i}\left(y_{i}, x_{i}\right)\right)_{i \in I}\right) .
$$

On the other hand,

$E Q_{d^{-1}, \Phi}(x, y)=\Phi\left(\left(d_{i}^{-1}\left(x_{i}, y_{i}\right)\right)_{i \in I}\right)=\Phi\left(\left(d_{i}\left(y_{i}, x_{i}\right)\right)_{i \in I}\right)$.

Therefore we have that

$$
E Q_{d, \Phi}^{-1}(x, y)=E Q_{d^{-1}, \Phi}(x, y)
$$

for all $x, y \in X=\prod_{i \in I} X_{i}$.

In order to obtain a version of Proposition 6, given $\mathbf{a}, \mathbf{b} \in \mathbb{R}_{I, \infty}^{+}$, we will denote by $\mathbf{a} \sqcup \mathbf{b}$ the element of $\mathbb{R}_{I, \infty}^{+}$defined by $(a \sqcup b)_{i}=a_{i} \vee_{\infty} b_{i}$ for all $i \in I$.

Proposition 13. Let $\Phi: \mathbb{R}_{I, \infty}^{+} \rightarrow \mathbb{R}_{\infty}^{+}$be an extended quasi-metric aggregation function and let $\left\{\left(X_{i}, d_{i}\right)\right\}_{i \in I}$ be an indexed family of extended quasimetric spaces. Then the following assertions are equivalent:

(1) The below inequalities are satisfied for all $x, y \in$ $X=\prod_{i \in I} X_{i}$ :

$$
E Q_{d, \Phi}^{s}(x, y) \leqslant_{\infty} E Q_{d^{s}, \Phi}(x, y) \leqslant_{\infty} 2 E Q_{d, \Phi}^{s}(x, y),
$$

where

$$
E Q_{d^{s}, \Phi}(x, y)=\Phi\left(\left(d_{i}^{s}\left(x_{i}, y_{i}\right)\right)_{i \in I}\right)
$$

for all $x, y \in X$.

(2) $\Phi$ is monotone.

Proof. (1) $\rightarrow$ (2). Let $\mathbf{a}, \mathbf{b} \in \mathbb{R}_{I, \infty}^{+}$such that $\mathbf{a} \preceq_{\infty} \mathbf{b}$. Take the indexed family of extended quasi-metric spaces $\left\{\left(X_{i}, d_{i}\right)\right\}_{i \in I}$ with $X_{i}=\left(\mathbb{R}_{\infty}^{+}\right)^{2}$ and $d_{i}=U_{\infty}$ for all $i \in I$. On the one hand, by Remark 1 , we have guaranteed the existence of $x_{a b}, y_{a b} \in X$ such that

$$
U_{\infty}\left(x_{a b_{i}}, y_{a b_{i}}\right)=a_{i} \text { and } U_{\infty}^{-1}\left(x_{a b_{i}}, y_{a b_{i}}\right)=b_{i}
$$

for all $i \in I$. Hence

$$
\Phi(\mathbf{a})=\Phi\left(\left(U_{\infty}\left(x_{a b_{i}}, y_{a b_{i}}\right)\right)_{i \in I}\right)=E Q_{d, \Phi}\left(x_{a b}, y_{a b}\right)
$$

and

$$
\Phi(\mathbf{b})=\Phi\left(\left(U_{\infty}^{-1}\left(x_{a b_{i}}, y_{a b_{i}}\right)\right)_{i \in I}\right)=E Q_{d, \Phi}^{-1}\left(x_{a b}, y_{a b}\right) .
$$

On the other hand, we have that

$$
\begin{aligned}
E Q_{d, \Phi}^{s}\left(x_{a b}, y_{a b}\right) & =E Q_{d, \Phi}\left(x_{a b}, y_{a b}\right) \vee_{\infty} E Q_{d, \Phi}^{-1}\left(x_{a b}, y_{a b}\right) \\
& =\Phi(\mathbf{a}) \vee_{\infty} \Phi(\mathbf{b})
\end{aligned}
$$

and that

$E Q_{d^{s}, \Phi}\left(x_{a b}, y_{a b}\right)=\Phi\left(\left(U_{\infty}^{s}\left(x_{a b_{i}}, y_{a b_{i}}\right)\right)_{i \in I}\right)=\Phi(\mathbf{a} \sqcup \mathbf{b})$.

Since $E Q_{d, \Phi}^{s}\left(x_{a b}, y_{a b}\right) \leqslant_{\infty} E Q_{d^{s}, \Phi}\left(x_{a b}, y_{a b}\right)$ we deduce that

$$
\Phi(\mathbf{a}) \leqslant_{\infty} \Phi(\mathbf{a}) \vee_{\infty} \Phi(\mathbf{b}) \leqslant_{\infty} \Phi(\mathbf{a} \sqcup \mathbf{b})=\Phi(\mathbf{b}) .
$$

So $\Phi$ is monotone.

$(2) \rightarrow(1)$. Let $x, y \in X$. We have that

$$
\begin{aligned}
E Q_{d, \Phi}^{s}(x, y) & =E Q_{d, \Phi}(x, y) \vee_{\infty} E Q_{d, \Phi}^{-1}(x, y) \\
& =\Phi\left(\left(d_{i}\left(x_{i}, y_{i}\right)\right)_{i \in I}\right) \vee_{\infty} \Phi\left(\left(d_{i}\left(y_{i}, x_{i}\right)\right)_{i \in I}\right) .
\end{aligned}
$$


Moreover,

$$
\begin{aligned}
E Q_{d^{s}, \Phi}(x, y) & =\Phi\left(\left(d_{i}^{s}\left(x_{i}, y_{i}\right)\right)_{i \in I}\right) \\
& =\Phi\left(\left(d_{i}\left(x_{i}, y_{i}\right)\right)_{i \in I} \vee_{\infty}\left(d_{i}\left(y_{i}, x_{i}\right)\right)_{i \in I}\right)
\end{aligned}
$$

Since $\Phi$ is monotone we deduce that

$$
\Phi\left(\left(d_{i}\left(x_{i}, y_{i}\right)\right)_{i \in I}\right) \leqslant \infty E Q_{d^{s}, \Phi}(x, y)
$$

and

$$
\Phi\left(\left(d_{i}\left(y_{i}, x_{i}\right)\right)_{i \in I}\right) \leqslant \infty E Q_{d^{s}, \Phi}(x, y) .
$$

Consequently $E Q_{d, \Phi}^{s}(x, y) \leqslant_{\infty} E Q_{d^{s}, \Phi}(x, y)$.

By statement (1) in Theorem 11 we have that

$$
\begin{aligned}
E Q_{d^{s}, \Phi}(x, y) & \leqslant \infty \Phi\left(\left(d_{i}\left(x_{i}, y_{i}\right)\right)_{i \in I}\right)+\Phi\left(\left(d_{i}\left(y_{i}, x_{i}\right)\right)_{i \in I}\right) \\
& =E Q_{d, \Phi}(x, y)+E Q_{d^{-1}, \Phi}(x, y) \\
& \leqslant \infty 2 E Q_{d, \Phi}^{s}(x, y)
\end{aligned}
$$

since $d_{i}^{s}\left(x_{i}, y_{i}\right) \leqslant_{\infty} d_{i}\left(x_{i}, y_{i}\right)+d_{i}\left(y_{i}, x_{i}\right)$ for all $i \in I$.

Combining both inequalities we conclude that

$$
E Q_{d, \Phi}^{s}(x, y) \leqslant_{\infty} E Q_{d^{s}, \Phi}(x, y) \leqslant_{\infty} 2 E Q_{d, \Phi}^{s}(x, y) .
$$

Since the preceding inequalities hold for all $x, y \in X$ we obtain the desired conclusion.

\section{Extended Quasi-metric Aggregation Functions and Quasi-metric Aggregation Functions: the Relationship}

A natural question that one can wonder is which is the relationship between extended quasi-metric aggregation functions and quasi-metric aggregation functions. We end the paper discussing which are the the extended quasi-metric aggregation functions that are simultaneously quasi-metric aggregation functions.

The next example shows that there exist extended quasi-metric aggregation functions which are not quasi-metric aggregation functions.

Example 4. Let $I=\{1\}$ and consider the function $\Phi$ introduced in Example 2. It is clear that $\Phi(u(0,1))=\Phi(1)=\infty$ and, thus, that $E Q_{u, \Phi}$ is not a quasi-metric. It follows that $\Phi$ is not a quasi-metric aggregation function.
Motivated by the preceding example we introduce a new class of extended quasi-metric aggregation functions which merge quasi-metrics into quasi-metrics and provide as output extended quasimetrics strictly in the case of the indexed family to merge is formed by some extended quasi-metrics that are not quasi-metrics.

Definition 2. A function $\Phi: \mathbb{R}_{I, \infty}^{+} \rightarrow \mathbb{R}_{\infty}^{+}$is a strict extended quasi-metric aggregation function if the function $E Q_{d, \Phi}: X \times X \rightarrow \mathbb{R}_{\infty}^{+}$is a (n extended) quasi-metric for every indexed family of (extended) quasi-metric spaces $\left\{\left(X_{i}, d_{i}\right)\right\}_{i \in I}$, where $X=\prod_{i \in I} X_{i}$ and

$$
E Q_{d, \Phi}(x, y)=\Phi\left(\left(d_{i}\left(x_{i}, y_{i}\right)\right)_{i \in I}\right)
$$

for all $x, y \in X$.

The below result characterizes the extended quasi-metric aggregation functions which are also quasi-metric aggregation functions.

The next remark will be useful in the announced characterization.

Remark 3. Let $d_{\mathbb{R}^{2}}: \mathbb{R}^{2} \times \mathbb{R}^{2} \rightarrow \mathbb{R}^{+}$be the quasimetric given by

$$
d_{\mathbb{R}^{2}}(x, y)=\left[\left(y_{1}-x_{1}\right) \vee 0\right]+\left[\left(y_{2}-x_{2}\right) \vee 0\right]
$$

for all $x=\left(x_{1}, x_{2}\right), y=\left(y_{1}, y_{2}\right) \in \mathbb{R}^{2}$. It is a simple matter to check that given $a, b, c \in \mathbb{R}^{+}$with $a \leqslant b+c$, there exist $x_{a b c}, y_{a b c}, z_{a b c} \in \mathbb{R}^{2}$ such that $d_{\mathbb{R}^{2}}\left(x_{a b c}, y_{a b c}\right)=a, \quad d_{\mathbb{R}^{2}}\left(x_{a b c}, z_{a b c}\right)=b$ and $d_{\mathbb{R}^{2}}\left(z_{a b c}, y_{a b c}\right)=c$. Concretely $x_{a b c}, y_{a b c}, z_{a b c} \in \mathbb{R}^{2}$ are given as follows:

If $a \leqslant b$, then $x=\left(\frac{-a}{2}, b+c\right), y=\left(\frac{a}{2}, c\right)$ and $z=\left(\frac{-a}{2}+b, 0\right)$.

If $b<a$, then $x=\left(\frac{-a}{2}, 0\right), y=\left(\frac{a}{2}, 0\right)$ and $z=$ $\left(\frac{-a}{2}+b, a-b-c\right)$.

Theorem 14. Let $\Phi: \mathbb{R}_{I, \infty}^{+} \rightarrow \mathbb{R}_{\infty}^{+}$be an extended quasi-metric aggregation function. Then the following statements are equivalent:

(1) $\Phi$ is a strict extended quasi-metric aggregation function.

(2) $\Phi(\boldsymbol{a})=\infty \Leftrightarrow a_{i}=\infty$ for some $i \in I$.

(3) $\Phi$ is monotone and $\operatorname{Im}\left(\left.\Phi\right|_{\mathbb{R}_{I}^{+}}\right) \subseteq \mathbb{R}^{+}$. 
Proof. (1) $\rightarrow$ (2). For the purpose of contradiction we suppose that there exists $\mathbf{x} \in \mathbb{R}_{I, \infty}^{+}$such that $\Phi(\mathbf{a})=\infty$ and $a_{i}<\infty$ for all $i \in I$. Since $a_{i}<\infty$ we obtain that $u\left(0, a_{i}\right)=a_{i}$ for all $i \in I$.

Next consider the indexed family of quasi-metric spaces $\left\{\left(X_{i}, d_{i}\right)\right\}_{i \in I}$ with $X_{i}=\mathbb{R}^{+}$and $d_{i}=u$ for all $i \in I$. Then the function $Q_{d, \Phi}$ given, for all $x, y \in X=\prod_{i \in I} X_{i}$, by

$$
Q_{d, \Phi}(x, y)=\Phi\left(\left(d_{i}\left(x_{i}, y_{i}\right)\right)_{i \in I}\right)
$$

is a quasi-metric. Whence we deduce that $Q_{d, \Phi}(x, y) \neq \infty$ for all $x, y \in X$. However, taking $x \in X$ with $x_{i}=a_{i}$, we have that

$$
\infty=\Phi(\mathbf{a})=\Phi\left(\left(u\left(0, a_{i}\right)\right)_{i \in I}\right)=Q_{d, \Phi}(0, x) \neq \infty,
$$

which is a contradiction.

$(2) \rightarrow$ (3). Obviously $\operatorname{Im}\left(\left.\Phi\right|_{\mathbb{R}_{I}^{+}}\right) \subseteq \mathbb{R}^{+}$. It remains to prove that $\Phi$ is monotone. To this end, consider $\mathbf{a}, \mathbf{b} \in \mathbb{R}_{I, \infty}^{+}$with $\mathbf{a} \preceq_{\infty} \mathbf{b}$. We distinguish two cases:

Case 1. $a_{i_{0}}=\infty$ for some $i_{0} \in I$. Then $b_{i_{0}}=\infty$, since $a_{i} \leqslant \infty b_{i}$ for all $i \in I$. Consequently $\Phi(\mathbf{a})=$ $\infty=\Phi(\mathbf{b})$.

Case 2. $a_{i}<\infty$ for all $i \in I$. Then we assume that $b_{i}<\infty$ for all $i \in I$ because otherwise we obtain immediately that $\Phi(\mathbf{a})<\infty=\Phi(\mathbf{b})$. Hence, by Remark 3, there exist $x_{a_{i} b_{i} c_{i}}, y_{a_{i} b_{i} c_{i}}, z_{a_{i} b_{i} c_{i}} \in \mathbb{R}^{2}$ such that $d_{\mathbb{R}^{2}}\left(x_{a_{i} b_{i} c_{i}}, y_{a_{i} b_{i} c_{i}}\right)=a_{i}, d_{\mathbb{R}^{2}}\left(x_{a_{i} b_{i} c_{i}}, z_{a_{i} b_{i} c_{i}}\right)=b_{i}$ and $d_{\mathbb{R}^{2}}\left(z_{a_{i} b_{i} c_{i}}, y_{a_{i} b_{i} c_{i}}\right)=0$.

Consider the indexed family of extended quasimetric spaces $\left\{\left(X_{i}, d_{i}\right)\right\}_{i \in I}$ with $X_{i}=\mathbb{R}^{2}$ and $d_{i}=$ $d_{\mathbb{R}^{2}}$ for all $i \in I$. Since $\Phi$ is an extended quasimetric preserving function, by statement (2), we have that $E Q_{d, \Phi}$ is a quasi-metric on $X=\prod_{i \in I} X_{i}$. Set $x_{a b c}, y_{a b c}, z_{a b c}$ the elements of $X$ such that $x_{a b c_{i}}=$ $x_{a_{i} b_{i} c_{i}}, y_{a b c_{i}}=y_{a_{i} b_{i} c_{i}}$ and $z_{a b c_{i}}=z_{a_{i} b_{i} c_{i}}$ for all $i \in I$. Then we obtain that

$$
\begin{aligned}
\Phi(\mathbf{a}) & =\Phi\left(\left(d_{\mathbb{R}^{2}}\left(x_{a_{i} b_{i} c_{i}}, y_{a_{i} b_{i} c_{i}}\right)\right)_{i \in I}\right) \\
& =E Q_{d, \Phi}\left(x_{a b c}, y_{a b c}\right) \\
& \leqslant E Q_{d, \Phi}\left(x_{a b c}, z_{a b c}\right)+E Q_{d, \Phi}\left(z_{a b c}, y_{a b c}\right) \\
& =\Phi\left(\left(d_{\mathbb{R}^{2}}\left(x_{a_{i} b_{i} c_{i}}, z_{a_{i} b_{i} c_{i}}\right)\right)_{i \in I}\right) \\
& +\Phi\left(\left(d_{\mathbb{R}^{2}}\left(z_{a_{i} b_{i} c_{i}}, y_{a_{i} b_{i} c_{i}}\right)\right)_{i \in I}\right) \\
& =\Phi(\mathbf{b})+\Phi(\mathbf{0})=\Phi(\mathbf{b}) .
\end{aligned}
$$

Therefore $\Phi$ is monotone.

(3) $\rightarrow$ (1). By Theorem 4, we have that the restriction of $\Phi$ to $\mathbb{R}_{I}^{+}$is a quasi-metric aggregation function and, thus, that $\Phi$ is a strict extended quasimetric aggregation function.

The next example shows that the new class of extended quasi-metric aggregation functions is not empty.

Example 5. Set $I=\{1,2, \ldots, n\} . \quad$ Fix $w_{1}, w_{2}, \ldots, w_{n} \in \mathbb{R}^{+}$and let $\Phi: \mathbb{R}_{I, \infty}^{+} \rightarrow \mathbb{R}_{\infty}^{+}$be the function given by $\Phi(\mathbf{x})=\sum_{i=1}^{n} w_{i} x_{i}$ for all $\mathbf{x} \in \mathbb{R}_{I, \infty}^{+}$. It is not hard to check that $\Phi$ is an extended quasimetric aggregation function. Moreover, condition (2) in statement of Theorem 14 gives, in addition, that $\Phi$ is a strict quasi-metric aggregation function.

Examples 2 and 3 provide two instances of extended quasi-metric aggregation functions that are not strict extended quasi-metric aggregations functions.

As a consequence of Theorems 11 and 14 we obtain the below result.

Corollary 15. Let $\Phi: \mathbb{R}_{I, \infty}^{+} \rightarrow \mathbb{R}_{\infty}^{+}$be an extended quasi-metric aggregation function. Then the following statements are equivalent:

(1) $\Phi$ is a strict extended quasi-metric aggregation function.

(2) $\Phi(\boldsymbol{x})=\infty \Leftrightarrow x_{i}=\infty$ for some $i \in I$.

(3) $\Phi$ is monotone and $\operatorname{Im}\left(\left.\Phi\right|_{\mathbb{R}_{I}^{+}}\right) \subseteq \mathbb{R}^{+}$.

(4) $\operatorname{Im}\left(\left.\Phi\right|_{\mathbb{R}_{I}^{+}}\right) \subseteq \mathbb{R}^{+}$and $\Phi(\boldsymbol{a}) \leqslant_{\infty} \Phi(\boldsymbol{b})+\Phi(\boldsymbol{c})$ for all $\boldsymbol{a}, \boldsymbol{b}, \boldsymbol{c} \in \mathbb{R}_{I, \infty}^{+}$with $\boldsymbol{a} \preceq_{\infty} \boldsymbol{b}+\boldsymbol{c}$.

Remark 4. Note that, by Proposition 13, each strict extended quasi-metric aggregation function $\Phi: \mathbb{R}_{I, \infty}^{+} \rightarrow \mathbb{R}_{\infty}^{+}$satisfies for every indexed family of extended quasi-metric spaces $\left\{\left(X_{i}, d_{i}\right)\right\}_{i \in I}$ and for all $x, y \in X=\prod_{i \in I} X_{i}$ the below inequalities:

$$
E Q_{d, \Phi}^{s}(x, y) \leqslant_{\infty} E Q_{d^{s}, \Phi}(x, y) \leqslant_{\infty} 2 E Q_{d, \Phi}^{s}(x, y) .
$$




\section{Conclusion}

In this paper we have continued the research line begun by J. Borsík, J. Doboš in ${ }^{1}$. Concretely, we have studied the problem of how to merge a (not necessary finite) collection of extended quasi-metrics in order to obtain a single one as output. Hence we have introduced the notion of extended quasimetric aggregation function, which extends the original notion given by Borsík and Doboš, and we have provided a description of such functions. Moreover, we have discussed what is the relationship between the problem of merging quasi-metrics, studied and solved by G. Mayor and O. Valero in 18 and ${ }^{19}$, and the extended quasi-metric aggregation one. Since extended quasi-metrics have been used in asymptotic complexity analysis of programs and algorithms $\left({ }^{10},{ }^{26}\right.$ and $\left.{ }^{27}\right)$ it seems natural to wonder if the aggregation theory exposed in the paper can be also applied to the aforesaid field. So, as further work, it seems interesting to focus our investigation on the possible utility of our proved results in the theory of computational complexity.

\section{Acknowledgments}

The authors acknowledge the support of the Spanish Ministry of Science and Innovation, grants MTM2009-10320, MTM2012-31847 and MTM2009-10962.

\section{References}

1. J. Borsík, J. Doboš, On a product of metric spaces, Math. Slovaca 31 (1981), 193-205.

2. A. Pradera, E. Trillas, E. Castiñeira, On distances aggregation, in: Proc. of the Information Processing and Management of Uncertainty in Knowledge-based Systems international Conference (2000), 693-700.

3. A. Pradera, E. Trillas, E. Castiñeira, On the aggregation of some classes of fuzzy relations, in: Technologies for constructing intelligent systems, B. BouchonMeunier, J. Gutierrez, L. Magdalena and R. Yager (Eds.), Springer-Verlag, 125-147, 2002.

4. A. Pradera, E. Trillas, A note on pseudometrics aggregation, Int. J. Gen. Syst. 31 (2002), 41-51.

5. J. Casasnovas, F. Roselló, Averaging fuzzy biopolymers, Fuzzy Set. Syst. 152 (2005), 139-158.
6. J. Casasnovas, F. Roselló, Midpoints as average representations of pairs of descriptions by means of fuzzy subsets, in: Proc. of the Information Processing and Management of Uncertainty in Knowledge-based Systems International Conference (2005), 2157-2164.

7. M. Schellekens, The Smyth completion: A common foundation for denonational semantics and complexity analysis, Electron. Notes Theor. Comput. Sci.1 (1995), 211-232.

8. L.M. García-Raffi, S. Romaguera, E.A. SánchezPérez, The supremum asymmetric norm on sequence algebras: A general framework to measure complexity spaces, Electron. Notes Theor. Comput. Sci.74 (2003), 39-50.

9. L.M. García-Raffi, S. Romaguera, E.A. SánchezPérez, Sequence spaces and asymmetric norms in the theory of computational complexity, Math. Comput. Model. 36 (2002), 1-11.

10. S. Romaguera, E. A. Sánchez-Pérez, O. Valero, Computing complexity distances between algorithms, Kybernetika, 39 (2003), 569-582.

11. L.M. García-Raffi, S. Romaguera, E.A. SánchezPérez, O. Valero, Normed semialgebras: a mathematical model for the complexity analysis of programs and algorithms, in: Proc. of the Seventh World Multiconference on Systemics, Cybernetics and Informatics (2003), vol. II, 55-58.

12. A. K. Seda, Quasi-metrics and the semantics of logic programs, Fund. Inform. 29 (1997), 97-117.

13. A. K. Seda, Some Issues Concerning Fixed Points in Computational Logic: Quasi-Metrics, Multivalued Mappings and the Knaster-Tarski Theorem, Topology Proc. 24 (1999), 223-250.

14. A. Stojmirović, Quasi-metric spaces with measure, Topology Proc. 28 (2004), 655-671.

15. A. Stojmirović, Quasi-metrics, similarities and searches: aspects of geometry of protein datasets, Ph.D. Thesis, Victoria University of Wellington, Wellington, 2005.

16. V. Pestov, A. Stojmirović, Indexing schemes for similarity search in datasets of short protein fragments, Inform. Syst. 35 (2007), 1145-1165.

17. A. Stojmirović, Y.-K. Yu, Geometric aspects of biological sequence comparison, J. Comput Biol. 16 (2009), 579-610.

18. G. Mayor, O.Valero, Aggregating asymmetric distances in Computer Sciences, in Proc. of the 8th International FLINS Conference on Computational Intelligence in Decision and Control (2008), 477-482.

19. G. Mayor, O. Valero, Aggregation of asymmetric distances in Computer Science, Inform. Sci. 180 (2010), 803-812.

20. H.P.A. Künzi, Nonsymmetric distances and their associated topologies: About the origins of basic ideas in the area of asymmetric topology, in: Handbook of the 
History of General Topology, C.E. Aull and R. Lowen (Eds.), vol. 3, Kluwer Acad. Publ., 853-968, 2001.

21. J. Casasnovas, O. Valero, A connection between Computer Science and Fuzzy Theory: Midpoints and running time of computing, Mathware \& Soft Computing 15 (2008), 251-261.

22. P. Tirado, O. Valero, The average running time of an algorithm as a midpoint between fuzzy sets, Math. Comput. Model. 49 (2009), 1852-1868.

23. S. Massanet, O. Valero, New results on metric aggregation, in: Proc. of the 17th Spanish Conference on Fuzzy Technology and Fuzzy Logic-Estylf (2012), 558-563.

24. J. Martin, G. Mayor, O. Valero, On aggregation of normed structures, Math. Comput. Model. 54 (2011), 815-827.

25. J. Martin, G. Mayor, O. Valero, A fixed point theorem for asymmetric distances via aggregation functions, in: Proc. of the 6th International Summer School on Aggregation Operators (2011), 217-222.

26. S. Romaguera, O. Valero, On the structure of the space of complexity partial functions, Int. J. Comput. Math. 85 (2008), pp. 631-640.

27. S. Romaguera, M. P. Schellekens, O. Valero, The complexity space of partial functions: A connection between complexity analysis and denotational semantics, Int. J. Comput. Math. 88 (2011), pp. 1819-1829. 